\title{
Fighting the Dengue Virus
}

\author{
Aniqa Faraz ${ }^{1}$, Muhammad Aadil ${ }^{2}$, Muhammad Nabeel Shafqat ${ }^{3}$ \\ 1. Internal Medicine, Jacobs School of Medicine and Biomedical Sciences, University at Buffalo, State \\ University of New York, Buffalo, New York, Buffalo, USA 2. Department of Medicine, FMH College of \\ Medicine and Dentistry, NEWARK, USA 3. Department of Internal Medicine, University of Medical \\ Sciences "Serafin Ruiz De Zarate" Villa Clara (Ucmvc)
}

$\square$ Corresponding author: Aniqa Faraz, aniqafar@buffalo.edu

Disclosures can be found in Additional Information at the end of the article

\section{Abstract}

The incidence of dengue has been on the upsurge in the last decade. It has affected around onethird of the world's population living in endemic areas. It can be asymptomatic or may present with some specific symptoms. No control measures have proven beneficial to decrease the prevalence of this disease. The emergence of dengue vaccine has been a revolutionary hope in the future of patients affected with this disease. No doubt, this vaccine has its limitations and may do more harm than good, but with correct use, it can prove to be the most beneficial step taken in managing dengue so far.

Categories: Internal Medicine, Preventive Medicine, Infectious Disease Keywords: dengue virus, dengue vaccine

\section{Editorial}

Dengue is a mosquito-borne viral illness transmitted to humans by Aedes aegypti. This mosquito is most commonly found in the tropical and subtropical regions of the world. The global incidence of this virus has increased dramatically in the last decades placing more than one-third of the world population living in endemic areas at risk, with an annual incidence of 400 million cases [1].

There has been no evidence of a decline in the spread of dengue with dengue prevention and control programs as the endemic countries have limited resources and control measures are brought into effect only in epidemics. So the arrival of dengue virus vaccine is indispensable to prevent complications and death from dengue fever [2].

Received 04/27/2017

Review began 05/08/2017 Review ended 05/19/2017 Published 05/24/2017

(c) Copyright 2017

Faraz et al. This is an open access article distributed under the terms of the Creative Commons Attribution License CC-BY 3.0., which permits unrestricted use, distribution, and reproduction in any medium, provided the original author and source are credited.
Four distinct serotypes have been documented for causing dengue: dengue virus one (DENV-1), dengue virus two (DENV-2), dengue virus three (DENV-3), and dengue virus four (DENV-4). Infection by one serotype confers a life-long immunity to that virus, but a subsequent infection with another serotype causes a more severe dengue disease with a mortality rate of 1-20\% [2]. The patients infected with dengue virus may be asymptomatic or may present with a severe illness sometimes leading to death.

Symptomatic individuals may present with fever, rash, headache, pain behind the eyes, muscle aches, bone and joint pains, nausea, and vomiting [1]. The atypical manifestations of dengue include encephalopathy, hepatitis, hemolytic-uremic syndrome, myocarditis, acute respiratory distress syndrome, and myositis. The most common complications include hemorrhage and shock [3]. 
An effective dengue vaccine is an important strategy to effectively control the disease burden. Sanofi Pasteur vaccine is the only one that made it through phase III trials. It is a tetravalent chimeric vaccine with marked efficacy against serotype three and four. It is also known to be the safest one. According to the World Health Organization, in 2016 it was approved for ages 945 years (Brazil) and 9-60 years (Paraguay); three doses to be given six months apart [4-5].

Dengvaxia, the dengue vaccine, is formed by splicing four dengue serotypes with the yellow fever virus 17D genes. With phase III trial under investigation, after two years of this trial, it was proven that Dengvaxia has better efficacy in those already immune to one of the serotypes, but has less efficacy in those with no prior infection, questioning the usefulness of this vaccine in travelers to endemic areas. However, this vaccine significantly reduced the prevalence of mild and severe dengue and the rate of hospitalization in those previously affected. According to the Study on Global Ageing and Adult Health run by the World Health Organization, the greatest benefit is achieved in seropositive patients at the time of vaccination.

\section{Expert opinion}

The goal of eradication of the epidemic of dengue can only be achieved by providing access to the low-cost vaccine in an abundant supply to endemic countries and implementing the appropriate measures of control like an effective water supply system, waste disposal, and the use of protective clothing and insecticide spray in travelers to these regions.

No doubt, dengue vaccine is a new hope to combat the illness and hospitalization caused by dengue, but its use is limited to places where it will do more good than harm.

There is still uncertainty about this vaccine and more research needs to be done on the dose and the selection of recipients for this vaccine. Its effectiveness depends on a number of factors including the age of the patient receiving the vaccine, the type of dengue, previous infection with dengue, and the local epidemiology.

The vaccine will win the battle if serological tests are done before the use of the vaccine. The correct use of this vaccine in future will decrease the number of severe cases and hospitalizations leading to a significant achievement.

\section{Additional Information}

\section{Disclosures}

Conflicts of interest: In compliance with the ICMJE uniform disclosure form, all authors declare the following: Payment/services info: All authors have declared that no financial support was received from any organization for the submitted work. Financial relationships: All authors have declared that they have no financial relationships at present or within the previous three years with any organizations that might have an interest in the submitted work. Other relationships: All authors have declared that there are no other relationships or activities that could appear to have influenced the submitted work.

\section{References}

1. Ayukekbong JA, Oyero OG, Nnukwu SE, et al.: Value of routine dengue diagnosis in endemic countries. World J Virol. 2017, 6:9-16.

2. Torres JR, Orduna TA, Piña-pozas M, et al.: Epidemiological characteristics of dengue disease in Latin America and in the Caribbean: a systematic review of the literature. J Trop Med. 2017, 2017:18. 10.1155/2017/8045435

3. Pawaria A, Mishra D, Juneja M, et al.: Atypical manifestations of dengue fever . Indian Pediatr. 2014, 51:495-497. 10.1007/s13312-014-0434-8 
Cureus

4. Schwartz LM, Halloran ME, Durbin AP, et al.: The dengue vaccine pipeline: implications for the future of dengue control. Vaccine. 2015, 33:3293-3298. 10.1016/j.vaccine.2015.05.010

5. Godoi IP, Santos AS, Reis EA, et al.: Consumer willingness to pay for dengue vaccine (CYDTDV, Dengvaxia R ) in Brazil; implications for future pricing considerations. Front Pharmacol. 2017, 8:1-9. 10.3389/fphar.2017.00041 